\title{
Development of a Vibration-Based Crack Diagnos- tic Application Using the MANFIS Technique
}

\author{
Amiya Kumar Dash \\ Department of Mechanical Engineering, I.T.E.R. Bhubaneswar, Orissa, India, 751030
}

\author{
Dayal R. Parhi \\ Department of Mechanical Engineering, N.I.T Rourkela, Orissa, India, 769008
}

\begin{abstract}
(Received September 20, 2010, Provisionally Accepted August 23, 2011, Accepted February 1, 2012)
This paper analyses the effectiveness of a newly developed, multiple-crack diagnostic tool in dynamic structures using the multiple adaptive neuro-fuzzy inference system (MANFIS) technique. The effect of crack characteristics on the vibration responses have been investigated with different boundary conditions using finite element and numerical analysis. The first three relative natural frequencies, the difference of the first three average relative mode shapes, crack locations, and crack depths are used to train the fuzzy and neural controllers of the MANFIS system. The MANFIS controller is comprised of an input layer, a hidden layer and an output layer. The fuzzy segment uses the first three relative natural frequencies and the difference of the first three average relative mode shapes as the inputs. The hidden layer processes the outputs from the fuzzy controller. Finally, relative crack locations and relative crack depths are outputs from the developed MANFIS controller. It is observed that the predicted values of relative crack locations and relative crack depths from the formulated technique are well in agreement with the results from experimental analysis. The proposed methodology demonstrates its capability to be a suitable non destructive technique for fault identification in vibrating structures.
\end{abstract}

\section{INTRODUCTION}

The presence of a transverse crack in shaft, rotor, and structures incurs a potential risk of destruction or collapse. This produces high costs of production and maintenance. Detection of a crack in its early stages may save the system for use after repair. By monitoring the system, depending upon the type and severity of the crack, it may be possible in some cases to extend the use of a flawed member without risking a catastrophic failure, while arrangements are being made for a replacement rotor.

Tada et al. ${ }^{1}$ have provided the basis for calculation of a compliance matrix for damage detection following fracture mechanics theory. Zheng et al. ${ }^{2}$ have presented a method based on the finite element method for detection of a crack in a faulty structural member. Narkis ${ }^{3}$ has stated thatthe crack can be simulated as an equivalent spring, connecting the two segments of the beam. Analysis of this approximate model results in algebraic equations that relate the natural frequencies of beam and crack characteristics. Yokoyama et al. ${ }^{4}$ have studied the vibration characteristics of a uniform Bernoulli-Euler beam with a single-edge crack using a modified line-spring model. A new method of vibration-based damage identification in structures exhibiting axial and torsional responses has been proposed by Duffey et al. ${ }^{5}$ An analysis has been performed by Patil et al. $^{6}$ for the detection of a crack using frequency measurements. Their method is based on transverse vibration modeling through the transfer matrix method. Identification of location and severity of damage in structures using frequency response function (FRF) data have been formulated by Hwang et al. ${ }^{7}$ A method for crack identification in cracked beams based on wavelet analysis has been presented by Loutridis et al. ${ }^{8}$ using a continuous wavelet transform. The location of the crack is determined by the sudden changes in the spatial variation of the transformed response. Dharmaraju et al. ${ }^{9}$ have used Euler-Bernoulli beam element and finite element modeling for crack identification. Khiem et al. ${ }^{10}$ have formulated a method to detect cracks of beams by analyzing natural frequencies in the form of a nonlinear optimization problem. Parhi et al. ${ }^{11}$ has designed a control system using fuzzy logic. Fuzzy rules embedded in the controller of a mobile robot enable it to avoid obstacles in a cluttered environment that includes other mobile robots. Fuzzy finite element method for static analysis of engineering systems has been done by Rao et al. ${ }^{12}$ using an optimization-based scheme taking fuzzy parameters into consideration. A fuzzy arithmetical approach has been used by Hanss et al. ${ }^{13}$ for the solution of finite element problems involving uncertain parameters. Boutros et al. ${ }^{14}$ have proposed a simple, effective, and robust fusion approach based on fuzzy logic and a sugeno-style inference engine for condition monitoring. Haykin ${ }^{15}$ has presented the use of the neural network as a data processing tool for various applications. Kadi et al. ${ }^{16}$ have attempted to reflect on the work done in the mechanical modeling of fiber-reinforced composite materials using ANN during the last decade. Lucon et al. ${ }^{17}$ have utilized artificial neural networks in place of a traditional micromechanical approach to calculate the global elastic properties of composite materials given the local properties and local geometry. Singh et al. ${ }^{18}$ have reviewed the progress made in electrical drivecondition monitoring and diagnostic research and development in general, as well as in induction machine drive-condition monitoring and diagnostic research and development using expert systems, a neural network, and fuzzy logic. Xu et al. ${ }^{19}$ 